\title{
Comparing two trainable grammatical relations finders *
}

\author{
Alexander Yeh \\ Mitre Corp. \\ 202 Burlington Rd. \\ Bedford, MA 01730 \\ USA \\ asy@mitre.org
}

\begin{abstract}
Grammatical relationships (GRs) form an important level of natural language processing, but different sets of GRs are useful for different purposes. 'Therefore, one may often only have time to obtain a small training corpus with the desired GR annotations. On such a small training corpus, we compare two systems. They use different learning techniques, but we find that this difference by itself only has a minor effect. A larger factor is that in English, a different GR, length measure appears better suited for finding simple argument GRs than for finding modifier GRs. Wo also find that partitioning the data may help momory-based lcarning.
\end{abstract}

\section{Introduction}

Grammatical relationships (GRs), which include arguments (e.g., subject and object) and modifiers, form an important level of natural language processing. GRs in the sentence

Yesterday, my cat ate the food in the bowl.

include ate having the subject my cat, the object the food and the time modifier Yesterday, and the food having the location modifier in (the bowl).

However, different sets of GRs are useful for different purposes. For example, Ferro et al. (1999) is interested in semantic interpretation, and needs to differentiate between time, location and other modifiers. The SPARKLE project (Carroll et al., 1997), on the other hand,

\footnotetext{
* This paper reports on work performed at the MITRE Corporation under the support of the MITRE Sponsored Research Program. Marc Vilain, Lynette Hirschman and Warren Greiff have helped make this work happen. Christine Doran and John Henderson provided helpful editing. Copyright (C)2000 The MITRE Corporation. All rights reserved.
}

does not differentiate between these types of modifiers. As has been mentioned by John Carroll (personal communication), this is fine for information retrieval. Also, having less differentiation of the modifiers can make it easier to find them (Ferro et al., 1999).

Unless the desired set of GRs matches the set already annotated in some large training corpus (e.g., the Buchholy et al. (1999) GR finder used the GRs annotated in the Penn Treobank (Marcus et al., 1993)), one will have to either manually write rules to find the GRs or annotate a training corpus for the desired set. Manually writing rules is expensive, as is annotating a large corpus. We have performed experiments on learning to find GRs with just a small annotated training sot. Our starting point is the work described in Ferro et al. (1999), which used a fairly small training set.

This paper reports on a comparison between the transformation-based crror-driven larner described in Ferro et al. (1999) and the memory-based learner for GRs described in Buchloolz et al. (1999) on finding GRs to verbs ${ }^{1}$ by retraining the memory-based learnor with the data used in Ferro et al. (1999). We find that the transformation versus memory-based difference only seems to cause a small difference in the results. Most of the result differcnces seem to instead be caused by differences in the representations and information used by the learners. An example is that different GR length measures are used. In English, one measure seems better for recovering simple argument GRs, while another measure seems better for modifier GRs. We also find that partitioning the data sometimes helps memory-based learn-

\footnotetext{
${ }^{1}$ That is, GRs that have a verb as the relation target. For example, in Cats eat., there is a "subject" relation that has eat as the target and Cats as the source.
} 
ing.

\section{Differences Between the Two Systems}

Fiorro et al. (1999) and Buchholz et al. (1999) both describe learning systems to find GRs. The former (TR) uses transformation-based crror-driven learning (Brill and Resnik, 1994) and the latter (MB) uses memory-beased learning (Daclemans et al., 1999).

In addition, there are other differences. The I'R system includes several types of information not used in the MB system (some because memory-based systems have a harder time handling set-valued attributes): possible syntactic (Comlex) and semantic (Wordnet) classes of a chunk headword, the stem(s) and nimed-entity category (c.g., person, location), if any, of a dhunk headword, lexemes in a chunk besides the leadword, pl-attachment estimate and certain verb chunk properties (e.g., passive, infinitive).

Some lexemes (e.g., coordinating conjunctions and punctuation) are usually outside of any chunk. The TR system will store these in an attribute of the nearest chunk to the left and to the right of such a lexeme. The MP system represents such lexemes as if they are one word chunks. 'l'he MB system camnot use the 'T'R system mothod of storage because memorybased systems have difficulties with set-valued atitributes (value is 0 or more lexemes).

The MB system (and not the T'R system) also examines the number of commas and verb chunks crossed by a potential GR.

The space of possible GRs searched by the two systems is slightly different. 'L'le' 'IR system searches for Glis of length thiree cllunks or less. T'he MB system scarches for GRs which cross at most either zero (target to the source's left) or one (to the right) verb chunks.

Also, slightly different are the chunks examined relative to a potential GR. Both systems will examine the target and source clumks, plus the source's immediate neighboring chunks. The MB system also examines the source's second neighbor to the left. I'le' 'IR system instead also examines the target's immediate neighbors and all the chunks between the source and target.

The TR system has more data partitioning than the MB system. With the T'R system, possible GRs that have a different source chunk type (e.g., noun versus verb), or a different relationship type (c.g., sulject versus object) or dircction or length (in chunks) ase always considered separately and will be affected by different rules. The MB system will note such differences, but may decide to ignore some or all of them.

\section{Comparing the Two Systems}

\subsection{Experiment Set-Up}

One camnot directly compare the two systems from the descriptions given in Ferro et al. (1999) and Bucllhol\% et al. (1999), as the rosults in the descriptions were based on different, datia sets and on different assumptions of what is known and what needs to be found.

Hore wo test how woll the systems perform using the same small annotated training set, the 3299 words of elementary school reading comprehension test bodies used in Ferro et al. (1999). ${ }^{2}$ Wo are mainly interested in comparing the parts of the system that takes in syntax (nown, verl), etc.) chunks (also known as groups) and finds the GRs between those chunks. So for the experiment, we used the gencral TiMBL system (Daclemans et al., 19999) to just reconstruct the part of the MI3 system that takes in chunks and finds GRs. 'L'he input to bot] this reconstructed part and the 'TR system is data that has been manually annotatied for syntax chunks and GRs, along with automatic lexeme and sentence segmentation and part-ofspeech tagging. In addition, the TR system lias manual named-entity annotation, and antomatic estimations for verl properties and preposition and subordinate conjunction attachments (Ferro et al., 1999). Because the MB system was originally designed to handle GRs attached to verbs (and not noun to noun GRs, ctc.), wo ran the reconstructed part to only find GRs to verbs, and ignored other types of GRs when comparing the reconstructed part with the TR system. The test set is the 1151 word test set used in Ferro et al. (1999). Only GRs to verbs were exanined, so the eflective training set GR count fell from 1963 to 1298 and test set GI

\footnotetext{
${ }^{2}$ Note that if we had been trying to compare the two systems on a large annotated training set, the M]3 system would do botter by default just bocause the TR system would take too long to process a large training set.
} 
count from 748 to 500 .

\subsection{Initial Results}

In looking at the test set results, it is useful to divide up the GRs into the following sub-types:

1. Simple arguments: subject, object, indirect object, copula subject and object, expletive subject (c.g., "It" in "It rained today.").

2. Modifiers: time, location and other modifiers.

3. Not so simple arguments: arguments that syntactically resemble modifiers. These are location objects, and also subjects, objects and indirect objects that are attached via a preposition.

Neither system produces a spurious response for type 3 GRs, but neither system recalls many of the test keys cither. The reconstructed MB system recalls 6 of the 27 test key instances (22\%), the TR system recalls 7 (26\%). A possible explanation for these low performances is the lack of training data. Only $58(3 \%)$ of the waining data GR instances are of this type.

The type 2 GRs are another story. There are 103 instances of such GRs in the test set key. The results are

\begin{tabular}{|l|r|r|r|}
\hline & \multicolumn{3}{|c|}{ Type 2 GRs } \\
System & Recall & Precision & F-score \\
\hline MB & $47(46 \%)$ & $49 \%$ & $47 \%$ \\
\hline TR & $25(24 \%)$ & $64 \%$ & $35 \%$ \\
\hline
\end{tabular}

Recall is the number (and percentage) of the keys that are recalled. Precision is the number of correctly recalled keys divided by the number of GRs the system claims to exist. F-score is the harmonic mean of recall $(r)$ and precision $(p)$ percontages. It equals $2 p r /(p+r)$. Here, the differences in $r, p$ and F-score are all statistically significant. 3 The MB system performs better as measured by the F-score. But a trade-off is involved. The MB system has both a higher recall and a lower precision.

The bulk (370 or $74 \%$ ) of the 500 GR key instances in the test set are of type 1 and most

\footnotetext{
${ }^{3}$ When comparing differences in this paper, the statistical significance of the higher score being better than the lower score is tested with a one-sided test. Differences deemed statistically significant are significant at the $5 \%$ level. Differences deemed non-statistically significant are not significant at the $10 \%$ level.
}

of these are cither subjects or objects. With type 1. GRs, the results are

\begin{tabular}{|l|r|r|r|}
\hline & \multicolumn{3}{|c|}{ Type 1 GRs } \\
System & Recall & Precision & F-score \\
\hline MB & $231(62 \%)$ & $66 \%$ & $64 \%$ \\
\hline TR & $284(77 \%)$ & $82 \%$ & $79 \%$ \\
\hline
\end{tabular}

With these GRs, the 'TR system performs considerably better both in terms of recall and precision. Tho differences in all three scores are statistically significant.

Because $74 \%$ of the GR test key instances are of type 1, where the TR system performs better, this system performs bettor when looking at tho results for all the test GRs combined. Again, all three scoro differences aro statistically significant:

\begin{tabular}{|l|r|r|r|}
\hline & \multicolumn{3}{|c|}{ Combined Results } \\
System & Recall & Precision & F-score \\
\hline MB & $284(57 \%)$ & $63 \%$ & $60 \%$ \\
\hline TR & $316(63 \%)$ & $80 \%$ & $71 \%$ \\
\hline
\end{tabular}

Later, we tried some extensions of the reconstructed MB system to try to improve its overall result. We could improve the overall rosult by a combination of using the $I B I$ search algorithm (instead of IGTREE) in 'liMBL, rcstricting the potential GRs to those that crossed no verb chunks, adding estimates on preposition and complement attachments (as was done in 'I'R) and adding information on verb chunks about being passive, an infinitive or an unconjugated present participle. The overall F-scorc rose to $65 \%$ (63\% recall, $67 \%$ precision). 'T'his is an improvement, but the 'IR system is still better. The differences between these scores and the other MB and TR combined scores are statistically significant.

\subsection{Exploring the Result Differences}

\subsubsection{Type 2 GRs: modifiers}

The reconstructed $\mathrm{MB}$ system performs better at type 2 GRs. How can we account for this result difference?

Letting the TR system find longer GRs (beyond 3 chunks in length) does not help much. It only finds one more type 2 GR in the test set (adds $1 \%$ to recall and $1 \%$ or less to precision).

Rerumning the 'TR system rule learning with an information organization closer to the $M B$ system produces the same $47 \%$ F-score as the 
MI3 system (recall is lower, but precision is highor). Specifically, wo got this rosult whon the 'IlR system was lerun with no information on pp-atiachmonts, verb chunk poropertics (e.g., passive, infinitive), naned-entity labels or headword stems. Also, tho 'TR system low oxamines tho chmols oxaninod by the original Ml3

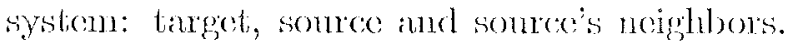
Ju ardation, instead of 6 absolute length categories (tiaged is 3 elnums to the ledt, 2 chunks, I chunk, and similarly for the right), the Cus considered now just fall into and anc partitioned inbo 3 rolative catogorics: target is the first verb chunk to the left, similarly to the right and larget is the secomel verts ohumk to the right, 'I'he

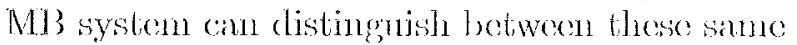
mative calcegorios.

Redoing this 'TR system roran without clumek hoadworel syntactic or semantic classes joroduces a $16 \%$ liscore. If in adelition, the ppoaltachnent, verb clnuk property, jamed-ntity label and headword stem information aro added back in, the l'-score actinally drops to $43 \%$. 'I'ho differences between these $47 \%, 46 \%$ and $13 \%$ rerun scores are not statistically significant.

So with typo 2 Gks, MBs system's bedted per formanes sooms to bo mainly due its ability to diflementiate the potential Gha by the featinte of

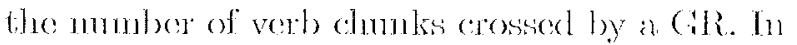
patticalat, making this and a few other changes wo the 'l'R systome increases its tescore to the

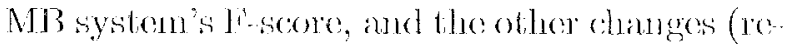
moving certatin information) does not have a sist nificant effect. So using the risht foatures can make a large difference.

For litese bype:2 Gles (modifiers) in Fin glish, it does secen that the mumber of vort clunks consed is a better way to goup pos. sible modifiens than tho absolnte chmol lemgth. An cxample is comparing I fly on Thesdey. and Ifly home from here on Tuesday. In both sentences, on I'uesday is a tiune moditiex of fly and on crosises no verts to reach fly (on atiaches to the first verlo to its left). But in the first sentence on is next to fly, while in the secound sontence, there are three clunuks separating on and fly.

\subsection{2 'Iype 1 GRs: simple arguments}

For type 1 (ths, the'lli system performs better. How can we account for this?

Much of the extra information the 'L'R systom examines (compareat to tho MB system) roos not secm to have much of an offect. When tho ITR system was rermu with no information on headword syntiatic on somantic classes, nemedentity labols or headword stems, the li-seore incrossed from $79 \%$ to $80 \%$. Another rerun that in addition lad no information on pp-atiachnont; estimates or any of the non-headwords in the dinnks also liad an F-scono of $80 \%$. A thinel rerun that furthermore had no information on verb chunk proferties (e.g., passive, infinitive) laad an I-score of $78 \%$. Th this set of li-scores, only the differences between the $80 \%$ scores and the $78 \%$ score are statistically significant.

Somo M13 system reruns sllowed factons that secmed to matiter nome. In the firsti resun, wo partitioned the data by potential (ald source clunk typo (e.g; nom volsus verb) and rall a separate memory based training and tost for

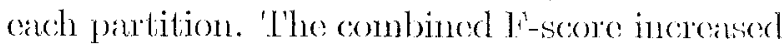
from $64 \%$ to $69 \%$. Aftorwards, wo male a rorum that rosombled the 'I'R system rom with the $78 \%$ l-scoro (cxcept that memory-based loarn ing was used): only Glis of length 3 chunks or: less were considened, tho datia was partitioned (in addition to somree chnnk ty])e) by (aR lemgti] and dincetion (e.g., lianget, is two chumks bo the

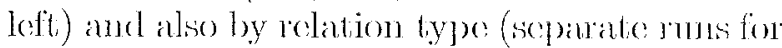

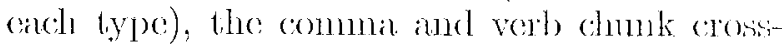
ing combts were not consirlered, and ti]e duntes normally oxamined by tho 'lle systom wore ex.

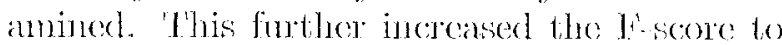
$75 \%$. Tn this sot of r-seores, all the differences wo statistically significant and all the F-scores in this sot are statistioally significantly different firom the 'l'R systen runs with the $78 \%$ and $79 \%$ li-scores.

From the statistically significant seove differances, it seoms that partitioning datia by po tential GR somec chunk type liolps (increase from $64 \%$ to $69 \%$ ), as does the rest of the partitioning performed and making some slight clanges in what is exannined (increase to $75 \%$ ), nsing transformation-based learning instead of memory-based learning (increase to $78 \%$ ) and using verl) chunk property information (increase to $80 \%)$

In the original MB system rum, the source chunk type and the potential GR lemgth and direction were already detemined by the memory-based learnor to be the most inportint 
attributes examined. So why would partitioning the data and runs by the values of these attributes be of extra help? A possible answer is that for different values, the relative order of importance of the other attributes (as determined by the memory-based learner) changes. For example, when the source chunk type is a noun, the second most important attribute is the source chunk's headword when the target is one to the right, but is the source chunk's right neighbor's headword when the target is one to the left. Partitioning the data and runs lets these different relative orders be used. Having one combined data set and run means that only one relative order is used. Note that while this partitioning may not be the standard way of using memory-based learning, it is consistent with the central idea in momory-based learning of storing all the training instances and trying to find the "nearest" training instance to a test case.

Another question is why using transformation-based (rulc) lcarning secms to be slightly better than memory-based learning for these typo 1 GRs. Memory-based learning kecps all of the training instances and does not try to find generalizations such as rules (Daclemans et al., 1999, Ch. 4). However, with type $1 \mathrm{GRs}$, a few simple generalizations can account for many of the instances. In the manner of Stevenson (1.998), we wrote a set of six simple rules that when run on the test set type 1 GRs produces an F-score of $77 \%$. 'This is better than what our reconstructed MB system originally achicved and is close to the 'TR system's original results (close enough not to be statistically significantily different). An example of these six rules: IF (1) the center chunk is a verb chunk and (2) is not considered as possibly passive and (3) its headword is not some form of to be and (4) the right neighbor is a noun or vorb chunk, ITHEN consider that chunk to the right as being an object of tho center chunk.

\section{Discussion}

GRs are important, but different sets of GRs are useful for different purposes. We have been looking at ways of improving automatic GR finders when one has only a small amount of data with the desired GR amnotations. In this paper, we compared a transformation rulebased system with a memory-based system on a small training corpus. We found that on GRs that point to verbs, most of the result differences can be accounted for by differences in the representations and information used. The type of GR determines which information is more important. The rule versus memory-based difference itself only seems to produce a small result; difference. We also find that partitioning the data may help memory-based learning.

\section{References}

E. Brill and P. Resnik. 1994. A rule-based approach to prepositional phrase attachment disambiguation. In 15th International Conf. on Computational Linguistics (COLING).

S. Buchholz, J. Veenstra, and W. Daelemans. 1999. Cascaded grammatical relation assignment. In Joint SIGDAT' Conference on Empirical Methods in NLP and Very Large Corpora (EMNLP/VLC'99). cs.CL/9906004.

J. Carroll, T. Briscoe, N. Calzolari, S. Federici, S. Montemagni, V. Pirrelli, G. Grefenstette, A. Sanfilippo, G. Carroll, and M. Rooth. 1997. Sparkle work package 1, specification of phrasal parsing, final report. Available at http://www.ilc.pi.cnr.it/sparkle/sparkle.htm, November.

W. Daclemans, J. Zavrel, K. van der Sloot, and A. van den Bosch. 1999. 'T'imbl: 'T'ilburg' menory based learner, version 2.0, roference guide. ILK Technical Report ILK 99-()1. Available from http://ilk.kub.nl/ ${ }^{i l k /-~}$ papers/ilk9901.ps.gz.

L. Ferro, M. Vilain, and $\Lambda$. Yoh. 1999. Learning transformation rules to find grammatical relations. In Computational natural language learning (CoNLL-99), pages 43-52. EACL'99 workshop, cs.CL/9906015.

M. Marcus, B. Santorini, and M. Marcinkiowicy. 1993. Building a large amnotated corpus of english: the penn trcebank. Computational Linguistics, 19(2).

M. Stevenson. 1998. Extracting syntactic relations using houristics. In I. KruijffKorbayová, editor, Proc. of the 3rd ESSLLI Student Scssion. Chapter 19. 